\title{
Detecting Signatures in Hyperspectral Image Data of Wounds: A Compound Model of Self-Organizing Map and Least Square Fitting
}

Redwan Abdo A. Mohammed, Daniel Schäle and Christoph Hornberger, Fakultät für Ingenieurwissenschaften, Hochschule Wismar* \{redwan.mohammed, daniel.schäle, christoph.hornberger \}@hs-wismar.de Steffen Emmert, Clinic for Dermatology and Venereology, University Medical Center Rostock, Rostock, Germany, steffen.emmert@med.uni-rostock.de

Hyperspectral imaging (HSI) has been used in measuring the physiological parameters of human tissue in many areas in clinical and emergency medicine, such as tissue perfusion measurements, wound analysis and flap monitoring, for diabetic foot syndrome and skin ulcers. Even though HSI measurements are often considered as an informative observable for the wounds' diagnostics, chronic wounds are still a major challenging problem in medicine.

The purpose of this study is to develop a method to discriminate spectral signatures in wound tissues. We have collected a training set of the intensity of the remitted light for different wound tissues from different patients using a TIVITA ${ }^{\mathrm{TM}}$ tissue camera (Diaspective Vision GmbH, Germany). We used a neural network technique (selforganizing map) to group areas with the same spectral properties together. The self-organizing map was an 8 by 6 hexagonal map with 48 neurons. The learned weights of each neuron represent a subset of similar spectral features in the training set. The results of this work indicate that neural network models are able to find clusters of closely related hyperspectral signatures in wound tissue, and thus can be used as a powerful tool to reach the anticipated classification. Moreover, we used a least square method to fit literature spectra (i.e. oxyhemoglobin $(\mathrm{O} 2 \mathrm{Hb})$, deoxyhemoglobin $(\mathrm{HHb})$, water and fat) to the different learned spectral classes. This procedure enables us to label each spectral class with the corresponding absorbance properties for the different absorbance of interest (i.e. O2Hb, $\mathrm{HHb}$, water and fat). The calculated parameters over a testing set were consistent with the expected behaviour and show a good agreement with the results of a second algorithm which was used with the TIVITA ${ }^{\text {TM }}$ tissue camera.

* This work is funded by the Europäischer Fonds für Regionale Entwicklung (EFRE) 


\section{Development of a mathematical model to calculate mechanical properties at the proximal end of peripheral vein catheters}

Leonard Pawelzik, FH Münster, Steinfurt, Germany, leonard.pawelzik@fh-muenster.de

Prof. Dr.-Ing. Claus Backhaus, FH Münster, Steinfurt, Germany, claus.backhaus@fh-muenster.de

Unintended administration of Infusion fluid into tissue forms extravasations. Those can be found in up to 6\% of all intensive care patients and up to $78 \%$ of preterm infants. Detection of beginning extravasation is currently not available.

In former research, a sensor was developed to detect the beginning of extravasation and to prevent patients from serious harms. The sensor applies a pressure pulse to the infusion line and measures pressure and flow trends at the peripheral vein catheter. Prospective, the mechanical properties of vein and tissue as well as the correct catheter placement are expected to be calculated by use of the step response.

A tissue simulator was build and validated to investigate influences of the mechanical properties of vein and tissue to the step response. It consists of an artificial blood flow and a test area including an artificial vein surrounded by gelatine to simulate human tissue.

The objective of research is the development of a mathematical model to describe the mechanical properties at the proximal end of a peripheral vein catheter as function of step response.

Mechanical properties in the test area are blood viscosity, -density, vein diameter, -compliance, -wall thickness, tissue density, -compliance and -inertia. Measurements are taken with different expressions of mechanical properties in accordance to the design of experiments principles for venous and extravasational catheter placement. A mathematical model is obtained by correlation analysis to describe the mechanical properties at the end of the catheter as function of step responses to the pressure pulse.

Mechanical properties at the end of the peripheral vein catheter vary from venous to extravasational placement. By applying the mathematical model, it's supposed that these can be calculated using the step response during infusion therapy. If changes in mechanical properties are detected, therapy can be stopped before extravasation can occur. 


\section{Verification of a Finite Element Updating Approach for in vivo Identification of the Nonlinear and Orthotropic Properties of Aortic Walls based on 4D Ultrasound Strain Imaging}

Andreas Wittek, Personalized Biomedical Engineering Laboratory, Frankfurt University of Applied Sciences, Frankfurt am Main, Germany, wittek@fb2.fra-uas.de

Wojciech Derwich, Vascular and Endovascular Surgery, Goethe University Hospital, Frankfurt am Main, Germany, Wojciech.Derwich@kgu.de

Thomas Schmitz-Rixen, Vascular and Endovascular Surgery, Goethe University Hospital, Frankfurt am Main, Germany, schmitz-rixen@em.uni-frankfurt.de

Claus-Peter Fritzen, Institute of Mechanics and Control Engineering - Mechatronics, University of Siegen, Germany, Claus-Peter.Fritzen@uni-siegen.de

Armin Huß, Personalized Biomedical Engineering Laboratory, Frankfurt University of Applied Sciences, Frankfurt am Main, Germany, huss@fb2.fra-uas.de

Christopher Blase, Personalized Biomedical Engineering Laboratory, Frankfurt University of Applied Sciences, Frankfurt am Main, Germany, cblase@fb2.fra-uas.de

Computational analysis of the biomechanics of the vascular system aims at a better understanding of its physiology and pathophysiology. To be of clinical use, however, these models have to be patient specific regarding geometry, boundary conditions and material. We present an in vivo approach to determining individual material properties of human aortae based on a new type of in vivo full field displacement data acquired by three-dimensional time resolved ultrasound (4DUS) imaging. We have developed a nested iterative Finite Element Updating method to solve two coupled inverse problems: The prestrains that are present in the imaged diastolic configuration of the aortic wall are determined. The solution of this problem is integrated in an iterative method to identify the parameters of a non-linear and orthotropic constitutive equation. In a numerical verification experiment, we have shown the feasibility of identifying nonlinear and orthotropic constitutive behaviour based on the observation of just two load cases, even though the load free geometry is unknown, if heterogeneous strain fields are available. Only clinically available 4D-US measurements of wall motion and diastolic and systolic blood pressure are required as input for the inverse FE updating approach. Application of the developed inverse approach to 4D-US data sets of three aortic wall segments from volunteers of different age and pathology resulted in the reproducible identification of three distinct and (patho-) physiologically reasonable constitutive behaviours. The use of patient-individual material properties in biomechanical modelling of AAAs is a step towards more personalized rupture risk assessment. 


\section{Hemolysis Prediction in Medical Devices Using Cell Deformation and Pore Formation Models}

Stefan Haßler, Chair for Computational Analysis of Technical Systems (CATS), CCES, RWTH Aachen University, 52056 Aachen, Germany, hassler@cats.rwth-aachen.de

Lutz Pauli, Chair for Computational Analysis of Technical Systems (CATS), CCES, RWTH Aachen University, 52056 Aachen, Germany, pauli@cats.rwth-aachen.de

Marek Behr, Chair for Computational Analysis of Technical Systems (CATS), CCES, RWTH Aachen University, 52056 Aachen, Germany, behr@cats.rwth-aachen.de

Ventricular Assist Devices (VADs) are nowadays used as a bridge-to-transplant, a bridge-to-recovery or even as an endstage therapy for cronically ill heart disease patients. These devices have to meet a certain hydraulic performance whilst being biocompatible.

Computational Hemodynamics is a valuable tool for the analysis of VADs, reducing the number of costly and time consuming experiments and giving insights in pump regions that are not accessible by the experiments. In order to give qualitative results, we believe that it is important to have a blood damage model that accounts for the physiological effects of the blood constituents. Our strain-based hemolysis model estimates the deformation of red blood cells (RBCs) in the blood flow with a so-called morphology model that predicts the relaxation, the elongation and the rotation of the RBCs. Eventually, pores will form on the surface of a deformed RBC and the size of these pores can be estimated with the minimization of an equation that accounts for the energy decrease through the reduction of stresses on the membrane under pore formation and the energy increase due to a higher exposure of the hydrophobic lipid bilayer to the blood plasma. With the total area of pores that form on the RBC's surface leading to hemoglobin leakage to the blood plasma, we compute the generation and distribution of the plasma-free hemoglobin.

We will present the aforementioned models for hemolysis estimation and discuss the associated numerical tools. Furthermore, we will show a comparison of the hemolysis results obtained from the simulation with experimental data for the benchmark blood pump of the U.S. Food and Drug Administration. 


\section{Temperature distribution and moisture wall film in the lung as effect of humidity and temperature of the respiratory air}

Bastian Schöneberger, Institute of fluid mechanics, Friedrich-Alexander University, Erlangen, Germany, bastian.schoeneberger@fau.de

Antonio Delgado, Insitute of fluid mechanics, Friedrich-Alexander University, Erlangen, Germany,

Antonio.delgado@fau.de

Naturally, the human body regulates the temperature and humidity of the air with the airway to the lung and the moisture wall film in the lung. This film is important to protect the lung against bacteria, particulars and respirable dust. In case of use a mechanical respirator, it is necessary to use a respirator gas humidifier. The effect of the conditioned air in the human lung is investigated with computational fluid dynamics (CFD). Transient simulations of the lung with different humidity and temperature of the respirator air were calculated.

The results shown that the moisture in the inhalation air has influence on air velocity, wall film, and temperature in the lung. Less humidity ensures a more homogeneous temperature and velocity distribution in the bronchial tree. Due to the uneven velocity distribution at higher humidity's in the respiratory air, a uniform temperature distribution is achieved only in higher generations of the lung. For the exhalation is this effect not important, because the air has the same temperature as the lung.

The wall film thickness is depending on the time step of the breath and the humidity of the respiratory air. During the exhalation, the thickness is decreasing and at the inhalation, the thickness is increasing. This thickness depends on the humidity of the inhalation air and temperature. The air moisture build the new wall film depending on the temperature gradient between air and lung. If the temperature of the respiratory air is higher than the lung temperature, the wall film can decreasing and if the temperature is lower with high moisture, the thickness of the wall film is increasing.

The effect of humidity and temperature of the respiratory air is important for the setting of a mechanical respirator to reduce inflammations and water in the lung. 


\section{Experimental and numerical investigations of fluid flow in bioreactors for optimized in vitro stem cell loading in xenografts}

Robert Ott: Institute for ImplantTechnology and Biomaterials e.V., Friedrich-Barnewitz-Str. 4, 18119 RostockWarnemünde, Germany, E-mail: robert.ott@uni-rostock.de

Carolin Wüstenhagen: Institute for ImplantTechnology and Biomaterials e.V., Friedrich-Barnewitz-Str. 4, 18119 Rostock-Warnemünde, Germany, E-mail: carolin.wuestenhagen@uni-rostock.de

Wolfram Schmidt: Institute for Biomedical Engineering, Friedrich-Barnewitz-Str. 4, 18119 Rostock-Warnemünde, Germany, E-mail: wolfram.schmidt@uni-rostock.de

Heiner Martin: Institute for Biomedical Engineering, Friedrich-Barnewitz-Str. 4, 18119 Rostock-Warnemünde, Germany, E-mail: heiner.martin@uni-rostock.de

Michael Stiehm: Institute for ImplantTechnology and Biomaterials e.V., Friedrich-Barnewitz-Str. 4, 18119 RostockWarnemünde, Germany, E-mail: michael.stiehm@uni-rostock.de

Annika Kasten: Department of Oral, Maxillofacial, and Plastic Surgery, Rostock University Medical Center, Schillingallee 35, 18057 Rostock, Germany, E-mail: annika.kasten@med.uni-rostock.de

Jan Liese: Department of Oral, Maxillofacial, and Plastic Surgery, Rostock University Medical Center, Schillingallee 35, 18057 Rostock, Germany, E-mail: jan.liese@med.uni-rostock.de

Carsten Fechner: Department of Oral, Maxillofacial, and Plastic Surgery, Rostock University Medical Center, Schillingallee 35, 18057 Rostock, Germany, E-Mail: Carsten.fechner@uni-rostock.de

Bernhard Frerich: Department of Oral, Maxillofacial, and Plastic Surgery, Rostock University Medical Center, Schillingallee 35, 18057 Rostock, Germany, bernhard.frerich@med.uni-rostock.de

Nadia Einnolf: RoweMed AG, Juri-Gagarin-Ring 4, 19370 Parchim, Germany, E-Mail: nadia.einnolf@rowemed.de

Niels Grabow: Institute for Biomedical Engineering, Friedrich-Barnewitz-Str. 4, 18119 Rostock-Warnemünde, Germany, E-Mail: niels.grabow@uni-rostock.de

Klaus-Peter Schmitz: Institute for ImplantTechnology and Biomaterials e.V., Friedrich-Barnewitz-Str. 4, 18119 Rostock-Warnemünde, Germany, E-Mail: klaus-peter.schmitz@uni-rostock.de

Stefan Siewert: Institute for ImplantTechnology and Biomaterials e.V., Friedrich-Barnewitz-Str. 4, 18119 RostockWarnemünde, Germany, E-Mail: stefan.siewert@uni-rostock.de

In tissue engineering and regenerative medicine mesenchymal stem cells (MSC) are widely used to replace and restore the function of dysfunctional or missing tissue. Recent studies have shown significant enhancements of the in vivo healing process following dentofacial bone augmentation procedures employing stem cell-loaded xenografts.

We conducted experimental and numerical investigations in perfusion flow bioreactor-xenograft-systems to identify flow conditions as well as bioreactor design features that allow for homogeneous MSC-distribution in Geistlich BioOss Block xenografts. Pressure gradient - velocity characteristics and flow distributions were investigated experimentally and numerically for two bioreactor designs at steady-state flow conditions with Reynolds numbers $(R e)$ ranging from $0.01 \leq R e \leq 0.32$. Distilled water at $20^{\circ} \mathrm{C}$ with a dynamic viscosity of $1.002 \mathrm{mPa} \cdot \mathrm{s}$ and a density of $998 \mathrm{~kg} / \mathrm{m}^{3}$ was used. The geometry of the xenograft utilized in three-dimensional computational fluid dynamics (CFD) simulation was obtained by means of micro-computed tomography $(\mu \mathrm{CT})$ at an isotropic spatial resolution of $9.5 \mu \mathrm{m}$. The permeability values calculated from the experimental data are in good accordance with the numerical results. The investigations showed that the increase of the inflow- and outflow-area diameter, as well as the decrease of the volumetric flow rate, result in a decreasing heterogeneity of the flow distribution within the xenograft. The calculated wall shear stress rates in the three-dimensional (3D) scaffold range from $1 \cdot 10^{-12} \mathrm{~Pa} \leq \tau \leq 0.2 \mathrm{~Pa}$. Experimentally validated CFD simulations introduced in this study provide an applicable tool to assess optimal flow conditions for homogeneous MSC distribution in bioreactor-xenograft-systems. 


\section{Expiratory flow control improves the homogeneity of pressure distribution in a four-compartment physical lung model}

Christin Wenzel, Department of Anesthesiology and Critical Care, Medical Center, University of Freiburg, Faculty of Medicine, Freiburg, Germany, christin.wenzel@uniklinik-freiburg.de

Carina Frey, Department of Anesthesiology and Critical Care, Medical Center, University of Freiburg, Faculty of Medicine, Freiburg, Germany, carina.frey@uniklinik-freiburg.de

Matthias Schneider, Department of Anesthesiology and Critical Care, Medical Center, University of Freiburg, Faculty of Medicine, Freiburg, Germany, matthias.schneider@uniklinik-freiburg.de

Stefan Schumann, Department of Anesthesiology and Critical Care, Medical Center, University of Freiburg, Faculty of Medicine, Freiburg, Germany, stefan.schumann@uniklinik-freiburg.de

Mechanical ventilation is a life-supporting key therapy. However mechanical ventilation can cause ventilator-induced lung-injury and post-operative complications. The passive expiration during conventional modes like volume controlled ventilation $(\mathrm{VCV})$ or pressure controlled ventilation (PCV) is associated with high expiratory flow rates and consequently rapid initial pressure drops. This may potentially promote inhomogeneous pressure distribution and high shear stress within an inhomogeneous lung. Controlling the flow during expiration is a new approach of ventilation therapy. Thereby, high flow rates and rapid pressure drops are limited. We hypothesized that ventilation modes with decelerated expiratory flow, like flow-controlled expiration (FLEX) and flow-controlled ventilation (FCV), enhance the homogeneity of pressure distribution compared to conventional ventilation modes.

For applying VCV and PCV we used a conventional ventilator (Evita 4, Dräger medical, Lübeck, Germany). FLEX was realized by VCV combined with a computer controlled resistance in the expiratory limb of the ventilation circuit . For the FCV mode we used the new type mechanical ventilator Evone (Ventinova Medical BV, Eindhoven, The Netherlands).

Therefore, we investigated the homogeneity of pressure distribution during VCV, PCV, FLEX and FCV in a fourcompartment physical lung model. Two compartments had a high compliance of $25 \mathrm{ml} / \mathrm{mbar}$ and two a low compliance of $10 \mathrm{ml} / \mathrm{mbar}$. They were respectively combined with either a high or a low resistance $(6.5 \mathrm{mbar} \cdot \mathrm{s} / 1 \mathrm{or} 2.8 \mathrm{mbar} \cdot \mathrm{s} / \mathrm{l})$. Pressure and flow rate were continuously measured within each compartment.

For each compartment we determined the time at which the expiratory pressure reached $50 \%$ of end-inspiratory pressure $\left(t_{50}\right)$ and calculated the maximal values of $t_{50}$ differences $\left(\Delta t_{50}\right)$ and the pressure difference $\left(\Delta p_{\max }\right)$ between all compartments during expiration. High values of $\Delta \mathrm{t}_{50}$ and $\Delta \mathrm{p}_{\max }$ correlate with a high inhomogeneity.

During VCV and PCV $t_{50}$ was lower than during FLEX and FCV, respectively (both $\mathrm{p}<0.001$ ). Overall, $\Delta \mathrm{t}_{50}$ was on average 3-fold higher and in specific situations even 6-fold higher during VCV and PCV (131 $\pm 20 \mathrm{~ms})$ than during FLEX and FCV $(35 \pm 6 \mathrm{~ms} ; \mathrm{p}<0.001)$. Further, the $\triangle$ pmax during VCV and PCV reached up to $3.8 \pm 0.2$ mbar but at maximum $0.6 \pm 0.1$ mbar during FLEX and FCV.

We conclude that the pressure distribution during expiration with FLEX and FCV is more homogeneous than with VCV and PCV. This is expected to reduce intrapulmonal shear stress and may thus be lung protective.

This project has received funding from the European Union's Horizon 2020 research and innovation program under grant agreement No. 691519. 